\title{
A IMPORTÂNCIA DO EXAME ODONTOLÓGICO PERIÓDICO OCUPACIONAL: UMA PROPOSTA DE PRONTUÁRIO ODONTOLÓGICO
}

THE IMPORTANCE OF OCCUPATIONAL DENTISTRY EXAMINATION: THE PROPOSE OF DENTISTRY PRONTUARY

\author{
Antonio Ricardo Borges Olival * \\ Senda Charone ** \\ Sonia Groisman ***
}

\begin{abstract}
RESUMO
A importância do exame periódico odontológico do trabalhador tem sido, até pouco tempo, negligenciada pelos empresários e órgãos reguladores. Atualmente se faz necessário um acompanhamento periódico Odontológico, uma vez que este se reveste de características importantes para o trabalhador e a empresa, permitindo uma atuaçáo ao trabalhador, relacionando seu ambiente de trabalho, seu estado psicológico e sua atividade na comunidade, com doenças profissionais, como lesôes irreversíveis provocadas pelo mau uso de equipamentos ou mesmo falha nas medidas de proteção, orientando assim o trabalho do CirurgiãoDentista para que o trinômio Agente Nocivo $<=>$ Meio Ambiente $<=>$ Trabalhador esteja perfeitamente equilibrado, objetivando diminuir o seu absenteísmo, aumentar o lucro do empresário. Para alcançar esse objetivo é necessário que se tenha em mãos a vida pregressa do funcionário, em seu local de trabalho, avaliar seu estado de saúde bucal através de uma anamnese e solicitar exames laboratoriais complementares ou específicos relacionados a seu estado de risco. A analise dos resultados obtidos orienta a tomada de medidas à proteção ou o encaminhamento ao tratamento que recupere a saúde do "objetivo principal" em questão, o trabalhador. O presente trabalho é dividido em duas etapas: uma revisão de literatura especializada em prontuários sobre saúde do trabalhador e uma proposta de prontuário odontológico especifico para a sua saúde.
\end{abstract}

DESCRITORES: Odontologia do trabalho - Registros odontológicos

\section{ABSTRACT}

The periodical dental exam for the workers has being since far negligenced by the employees and the legislation. Nowadays is important that periodical dentistry examination take place, once it contains important information it is for the worker and the company. It allayed to act together with the worker and the company, relating the work atmosphere and risks, in his psychological state as well aticivities in the community, professional diseases, like irreversible lesions promoted by the improper use of equipments, orientating the dental clinician towards the triangle: harmful agent= ecological surrounding= worker, would be perfectly equilibrated, in order to diminish the absence in the work. To achieve these goals it is necessary to record in the work environment the pre and present medical historical status. In this away it is possible to order specific laboratorial exams related to his risk health factors. The analysis of these results could oriented the prevention measures or the necessary treatment in order to restore the health of the principal goal; "the worker". The present work is divided in two parts, the first one is based in the specific review of literature and the second is the proposal on a specific dentistry protocol towards the work healthy.

DESCRIPTORS: Occupational dentistry - Dental records

\footnotetext{
* Especialista em Odontologia do Trabalho Associação Odontológica de São Cristóvão - RJ

** Especialista em Odontopediatria na Faculdade de Odontologia da Universidade Federal do Rio de Janeiro (FO/UFRJ) e Especialista em Odontologia em Saúde Coletiva FO/UFRJ

*** Profa Adjunto do Departamento de Odontologia Social e Preventiva da Universidade Federal do Rio de Janeiro
} 
Olival ARB, Charone S, Groisman S. A importância do exame odontológico periódico ocupacional: uma proposta de prontuário odontológico. Revista de Odontologia da Universidade Cidade de São Paulo 2008 jan-abr; 20(1):37-45

\section{INTRODUÇÃO E REVISÃO DE LITERATURA:}

Qualquer que seja a atividade econômica impulsionadora de um país em desenvolvimento, ela encontra no trabalho realizado pelo ser humano o instrumento viabilizador final dos objetivos pretendidos. Tal fato revela o interesse demonstrado pelas autoridades do país, em relação à saúde do trabalhador, qualquer que seja seu nível de trabalho. Há vários séculos, observadores verificaram uma íntima relação entre a atividade exercida pelo trabalhador e o tipo de doença que este adquiriu, gerando um interesse cada vez maior de proteção em seu ambiente de trabalho.

Domingues, em 2002, dissertando sobre o exame médico periódico, descreveu que foi na Inglaterra, em 1883, que se criou inicialmente a inspeção das oficinas e em 1842 a proibição de trabalho de mulheres e crianças em subterrâneos e, nesse mesmo ano, pela primeira vez, James Smith contrata um "médico de fábrica”. Em 1879 foi aprovada a primeira Lei Inglesa de Acidentes do Trabalho e em 1890 limitou-se a jornada de trabalho em 08 horas. Ainda segundo o autor, as sociedades desenvolvidas chegaram à compreensão de que a preservação da saúde e da integridade física do trabalhador, tanto proporciona seu bem-estar pessoal, familiar e social, quanto determina uma maior produtividade para a empresa, além de reduzir gastos com seguros e indenizaçóes previdenciárias. Com a experiência adquirida durante os anos, zelar pela saúde física e mental do trabalhador deixa de ser um ato de caridade, para assumir as feiçóes de dever social, na medida em que a prevenção aos acidentes e doenças do trabalho diminui o absenteísmo (ausência ao trabalho), colabora para o aumento da produção nacional além de reduzir gastos sociais previdenciários.

Em 25 de julho de 1972, com o decreto de no 70861, implantava-se o Programa Nacional de Valorização do Trabalhador (PNVT), que dentre outras atribuiçóes visava à formação de técnicos em higiene e segurança do trabalho (profissionais de nível superior e médio) e à realização de uma campanha de esclarecimento público das medidas de proteçáo contra os acidentes do trabalho.

No presente trabalho objetiva-se enfatizar a importância do Exame Odontológico Periódico, no Programa Médico de Saúde Ocupacional - PCMSO, pois acredita-se que seja de suma importância no controle da saúde do trabalhador e na sua interação com o meio ambiente, objetivando melhor qualidade de vida.

\section{a) Importância do Exame Odontológico Periódico}

Guimarães e Rocha ${ }^{4}$ (1979) definem a Odontologia do Trabalho como "a parte da Odontologia que trata de promover, preservar e reparar a saúde do trabalhador, conseqüente dos agravos, afecçóes ou doenças advindas do exercício profissional e que se manifestam na boca”, diferindo das demais especialidades por ter a sua ação diretamente dirigida e voltada para a prevenção de todos os agravos laborais, objetivando a prevenção de doenças conseqüentes da atuação profissional e dos acidentes do trabalho.

Segundo Silva et al. ${ }^{9}$ (1997), o tratamento odontológico deve iniciar-se com a anamnese. Esta deve ser bem conduzida, sendo norma técnica em qualquer situação clínica. As negligências na anamnese caracterizam erro profissional, podendo sujeitar o profissional a sançóes éticas e legais. Segundo esses autores, numa primeira consulta de atendimento clínico, as diferentes personalidades, ansiedade e o nível de conhecimento por parte dos pacientes podem perturbar o bom andamento da colheita de informações clínicas. O preconceito, constrangimento, o medo de rejeição ou mesmo a desinformação têm gerado situaçóes em que o paciente, conscientemente, omite informaçóes. Como o tratamento odontológico é muito específico, muitos pacientes acham que determinadas informaçóes não precisam ser relatadas ao dentista. "Por que o dentista, que vai apenas tratar de meus dentes, precisa saber que eu tenho AIDS?". Portanto, os odontólogos devem esclarecer os seus pacientes sobre a importância de terem acesso a todas as informaçóes clínicas para o bem da própria saúde do paciente e o sucesso da terapêutica odontológica.

Guimarães, citado por Oliveira et al. ${ }^{7}$ (1996), recomenda que se utilize um prontuário odontológico durante a anamnese, contendo o odontograma, sua identificação, a história clínica, o plano de tratamento, os exames complementares e, principalmente, todo o tratamento realizado. Outras questóes passíveis de serem esclarecidas pelo odonto-legista na Identificação Geral "postmortem", dizem respeito à estimativa da idade, do sexo, da estatura, a determinação do grupo étnico ou a cor da pele. Além de outras características como o diagnóstico de manchas ou líquidos provenientes da cavidade oral ou nela contida. Já a identificação individual distingue-se pela necessidade da presença de elementos comparativos anteriores à morte. Como por exemplo, em corpos carbonizados, os elementos dentários confrontados com os dados da ficha clínica odontológica anterior aos acontecimentos. 
Olival ARB, Charone S, Groisman S. A importância do exame odontológico periódico ocupacional: uma proposta de prontuário odontológico. Revista de Odontologia da Universidade Cidade de São Paulo 2008 jan-abr; 20(1):37-45

Segundo Silva et al. ${ }^{9}$ (1997), a competência do odonto-legista é múltipla, abrangendo a área civil, criminal, trabalhista e administrativa. $\mathrm{O}$ autor sugere ainda que a documentação em relação ao aspecto clínico do individuo tenha as características de um verdadeiro prontuário odontológico, que deve conter as seguintes partes:

1a) Anamnese: Para iniciar qualquer intervençáo em nosso paciente, há a necessidade de conhecer a saúde geral.

2a) Ficha Clínica: É uma parte integrante do prontuário odontológico, devendo apresentar informaçôes sobre o estado de saúde bucal do paciente, antes de iniciado o tratamento. Se for um especialista que recebeu um paciente, por encaminhamento de um colega, deve também dispor de todos os dados sobre o paciente na ficha clínica, para se resguardar de eventual responsabilidade, por atos operacionais não realizados ou de negligência profissional.

3a) Plano de Tratamento: Os limites de um tratamento na área de saúde são biológicos, muitas das vezes psicológicos, e também sociais e culturais. Portanto, é inadmissível a utilização do termo "orçamento", para definir as conseqüências precisas do ponto de vista clínico das fases de tratamento, muitas vezes imprevisíveis.

Os modelos de estudo ao lado de sua função clínica de colaborar no planejamento do tratamento podem também constituir elementos de prova judicial, portanto devem ser guardados. As radiografias, um dos exames complementares mais realizados pelo cirurgião-dentista, estão presentes na maioria dos processos, como matéria de prova.

De acordo com Mealey em 1996, descrevendo as implicaçóes periodontais e suas subseqüentes conseqüências para a saúde geral do paciente, o sucesso da terapia periodontal em um caso com complicações sistêmicas (diabetes mellitus) dá suporte ao conceito oferecido para a Comissão de Diabetes nos anos 70 - de que a doença periodontal deveria ser vista como a sexta maior complicação do diabetes mellitus.

Gomes e Magalhães ${ }^{5}$ (1980) sugerem a realização do Censo Bucal na empresa como recurso para aplicação de uma odontologia preventiva. Consiste no exame coletivo dos empregados, cujo objetivo é pesquisar manifestaçóes orais das moléstias profissionais, focos de etiologia dentária, exame da prevalência de cáries e periodontopatias, além de planejamento da terapêutica de maneira racional, sob o prisma de que o serviço odontológico da empresa deve visar por um lado à saúde oral do empregado e seu bem-estar e por outro representar o interesse econômico da empresa, mantendo o empregado são, apto a produzir mais e melhor, sem absenteísmo e obtendo-se com isto o retorno dos gastos na assistência prestada. Com o benefício da assistência odontológica o trabalhador não se ausentará da empresa, eliminando-se o problema do atestado, a procura pelo serviço público e pelo consultório particular.

Com essa revisão é possível perceber a grande importância que a anamnese criteriosa, com levantamento completo da história médica da paciente, possíveis medicamentos e doenças sistêmicas interrelacionadas levam a um diagnóstico mais específico e a um prognóstico melhorado nos tratamentos Odontológicos no que tange a saúde do trabalhador.

\section{b) Proposta de Prontuário Odontológico para Saúde do Trabalhador}

Cottone e Standish ${ }^{2}$ em 1992, em seu livro sobre Odontologia Forense, fazem referência às possibilidades de se verificar o prontuário odontológico nos casos de processos em que se avalia a responsabilidade profissional, servindo como meio de prova

Ramirez ${ }^{8}$ em 1992, descreve a importância de se registrarem os procedimentos realizados em cada paciente, até como forma de prevenção contra processos judiciais, pois registros clínicos bem elaborados permitirão esclarecer a atuação do profissional e definir se este executou os procedimentos e condutas corretas.

De acordo com Zimmermann et al. ${ }^{10} \mathrm{em}$ 1998, dissertando sobre a importância do prontuário odontológico, este pontua sobre suas questôes legais e éticas. No que tange a saúde do trabalhador, deve ser elaborado de maneira especifica para este fim. Primeiro por se tratar de matéria de caráter ético e legal no que diz respeito às normas para quem exerce a Odontologia e em segundo lugar para que se possa efetivamente garantir a saúde de trabalhador, eliminando-se os riscos ou prevenindo-os, evitando-se assim o agravo das doenças relacionadas a sua atividades profissionais.

O Conselho Federal de Odontologia, em 2004, elaborou uma proposta de "Prontuário Odontológico", para orientaçáo profissional, com o objetivo do cumprimento da exigência contida no inciso VIII do art. $5^{\circ}$ do Código de Ética Odontológica, que o artigo 5, versa sobre a elaboraçáo e manutenção de dados odontológicos atualizados nos prontuários dos pacientes, conserva- 
Olival ARB, Charone S, Groisman S. A importância do exame odontológico periódico ocupacional: uma proposta de prontuário odontológico. Revista de Odontologia da Universidade Cidade de São Paulo 2008 jan-abr; 20(1):37-45

dos em arquivos próprios. Nessa orientação profissional, descreve-se como obrigatório aos cirurgióes-dentistas, a manutençáo de toda a documentaçáo referente ao atendimento executado nos pacientes.

A presente monografia apresenta uma proposta de Prontuário Odontológico para Saúde do Trabalhador. (ANEXO I), devido à importância de ter documentos adequados, estruturados e devidamente arquivados, que contenham informações da saúde bucal do trabalhador, antes de sua admissão, periodicamente até a sua saída da empresa, e principalmente por terem caráter ético legal previsto no Código de Ética Odontológico em seu Art.5 Inciso VIII, e devido à pouca literatura especializada acerca de Prontuários de Saúde Bucal aplicados na odontologia do trabalho.

\section{DISCUSSÃO}

Após análise das contribuiçóes encaminhadas pelas entidades representativas da classe, dos coordenadores de curso de graduação e pós-graduação, bem como da breve revista da literatura, a comissão verificou que, diante da heterogeneidade do exercício da Odontologia no Brasil e dos constantes avanços da ciência odontológica, é extremamente difícil produzir um documento definitivo e estático.

Por outro lado, visando de forma semelhante à coexistência pacífica entre procedimentos reabilitadores odontológicos de elevada complexidade e o nível de assistência odontológica prestado pela rede pública de saúde, observa-se a necessidade de erigir um documento que atenda a táo distintos segmentos, merecendo destaque também as características de algumas especialidades, cujas peculiaridades não constituem regra comum no exercício da clínica geral odontológica, que constitui o contingente mais expressivo de profissionais clinicamente ativos.

Ainda, em face dos avanços tecnológicos, estão sendo apresentadas, em título à parte, consideraçóes sobre os arquivos digitais.

Dessa forma, o modelo proposto visa atender a vontade detectada no site do Conselho Federal de Odontologia, onde $97 \%$ dos profissionais participantes manifes- taram o desejo de receber um modelo de prontuário que possa, desde que corretamente preenchido, resguardar os profissionais da Odontologia em questóes éticas e legais.

Diante do exposto e amparada na sistematização do trabalho desenvolvido, a comissão entende que a terminologia Prontuário Odontológico é a que melhor atende, nos dias atuais, a designação do conjunto de documentos padronizados, ordenados e concisos, destinados ao registro dos cuidados odontológicos prestados ao paciente (Silva9 1997 e Zimmermann et al. ${ }^{10}$ 1998).

Da definição e do objetivo a que este trabalho se propôs depreende-se a necessidade de serem listados quais são e como devem ser elaborados esses documentos, os quais a comissão dividiu, didaticamente, em Documentos Fundamentais e Documentos Suplementares. São documentos fundamentais aqueles que deverão ser preenchidos em todo e qualquer atendimento ao paciente, sendo constituído da Ficha Clínica

\section{CONCLUSÕES}

O exame médico periódico representa para o Médico do Trabalho uma ferramenta importante na avaliação da saúde ocupacional do trabalhador, já que através dele pode-se verificar se as medidas de proteçáo recomendadas aqui; e no Programa Médico de Controle de Saúde Ocupacional - PCMSO, surtiram o efeito desejado, no fator mais importante que engloba a relação "médica trabalhador", que é a prevenção da doença profissional ou doença do trabalho, objetivo real da atividade desenvolvida por essa especialidade.

Se no PCMSO são identificadas as condições de riscos nocivos a que será submetido o trabalhador e se propóem medidas de proteção, seja com o uso de EPI Equipamento de Proteção Individual ou mesmo através de barreiras que impeçam o agente nocivo de entrar em contacto com ele, é através do Exame Periódico que se controla a sua saúde "física e psicológica", onde se podem analisar as alteraçóes dos vários parâmetros utilizados na avaliação, utilizando-se para tanto uma anamnese, exame clínico e exames laboratoriais de rotina e específicos, orientados para cada risco a que está exposto o trabalhador. 
Olival ARB, Charone S, Groisman S. A importância do exame odontológico periódico ocupacional: uma proposta de prontuário odontológico. Revista de Odontologia da Universidade Cidade de São Paulo 2008 jan-abr; 20(1):37-45

\section{INQUÉRITO DE SAÚDE}

\begin{tabular}{|c|c|c|c|}
\hline Anemia & Sim & Não & Não sei \\
\hline Hepatite & Sim & Não & Não sei \\
\hline Sífilis & Sim & Não & Não sei \\
\hline HIV & Sim & Não & Não sei \\
\hline Tuberculose & Sim & Não & Não sei \\
\hline Asma & Sim & Não & Não sei \\
\hline Fumante & Sim & Não & Não sei \\
\hline Hormônios & Sim & Não & Não sei \\
\hline Alcoolista & Sim & Não & Não sei \\
\hline Tatuagens & Sim & Não & Não sei \\
\hline Herpes/Aftas & Sim & Não & Não sei \\
\hline Gravidez & Sim & Não & Não sei \\
\hline Desmaios & Sim & Não & Não sei \\
\hline Febre Reumática & Sim & Não & Não sei \\
\hline Diabetes & Sim & Não & Não sei \\
\hline Epilepsia & Sim & Não & Não sei \\
\hline Cicatrização ruim & Sim & Não & Não sei \\
\hline Distúrbios Psico & Sim & Não & Não sei \\
\hline Endocardite Bact. & Sim & Não & Não sei \\
\hline Problema Hepático & Sim & Não & Não sei \\
\hline Problema Renal & Sim & Não & Não sei \\
\hline Problema Cardíaco & Sim & Não & Não sei \\
\hline Tensão Arterial & Sim & Não & Não sei \\
\hline Cirurgia & $\operatorname{Sim}$ & Não & Não sei \\
\hline Tumor & Sim & Não & Não sei \\
\hline Internação Hospital & Sim & Não & Não sei \\
\hline
\end{tabular}

Você possui alguma doença / problema significativo não mencionado? Sim ( ) Não ( )

Qual ?

Declaro que as informaçôes fornecidas são verdadeiras.

Data ,

Assinatura do funcionário 
Olival ARB, Charone S, Groisman S. A importância do exame odontológico periódico ocupacional: uma proposta de prontuário odontológico. Revista de Odontologia da Universidade Cidade de São Paulo 2008 jan-abr; 20(1):37-45

\section{HÁBITO}

- Roer unhas $(\mathrm{S})(\mathrm{N})$

- Respirar pela boca $(\mathrm{S})(\mathrm{N})$

- Tomar chimarrão $(\mathrm{S})(\mathrm{N})$

- Chupar dedo (S) (N)

- Morder caneta / lápis (S) (N)

- Ranger os dentes dia / à noite $(\mathrm{S})(\mathrm{N})$

- Outros :

\section{HIGIENE BUCAL}

- Fio / fita dental (S) (N)

- Escova interdental (S) (N)

- Escova, ( macia) ( média) (dura)

- Escova, ( unitufo ) ( bitufo ) (palito )

- Qual creme dental:

- Flúor para bochechos $(\mathrm{S})(\mathrm{N})$

- Costuma escovar a língua (S) (N)

-Outros bochechos?

\section{DIETA}

- Ingere alimentos / bebidas entre as refeições ? não sim :

\section{EXAME EXTRA-ORAL}

- Erupçóes:

- Nódulos:

- Musculatura:

- Manchas:

- Gânglios:

- Cicatrizes:

- Assimetrias:

- Glândulas salivares:

Exames Laboratoriais:

\section{Exames Radiológicos:}

\section{EXAME ORAL}

- Lábios:

- Bochechas:

- Língua:

- Base da língua:

- Assoalho bucal:

- Palato:

- Freios e bridas:

- Atrição/Abrasão/Erosão:

- Dentes supra numerários:

- Dentes retidos:

- Diastemas:

- Fluxo salivar:

- Oclusão:

- Hipoplasia de esmalte:

- Manchas:

- Mobilidade dental:

- Gengivas:

- Calculo/tártaro:

- Retração gengival:

Outros

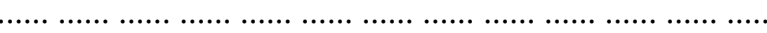

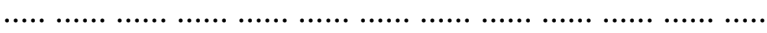

\section{Relatório Exame:}

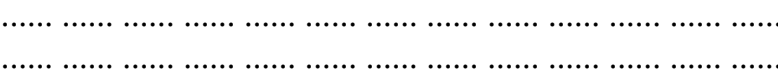

Cirurgião-Dentista responsável:

CRO:

Data:

Assinatura e Carimbo 


\section{ANEXO I \\ PRONTUÁRIO ODONTOLÓGICO- EXAME PERI- ÓDICO}

Leia com atenção antes de responder. As informaçóes que serão solicitadas no questionário abaixo fazem parte de seu exame periódico. Devido à necessidade de avaliarmos melhor sua condiçáo clinica, ressaltamos a importância da precisão das informaçôes. As informaçóes fornecidas são de sigilo deste departamento.

\section{FICHA DE EXAME ODONTOLÓGICO PERIÓDI- $\mathrm{CO}$}

Nome completo:

Função: $\ldots \ldots \ldots \ldots \ldots \ldots \ldots \ldots \ldots \ldots \ldots \ldots \ldots \ldots$

$\begin{array}{lllllllllllllllll}\operatorname{Sex0}(\ldots & \ldots & \ldots & \ldots & \ldots & \ldots & \ldots & \ldots & \ldots & \ldots & \ldots & \ldots & \ldots & \ldots & \ldots & \ldots\end{array}$ Data de admissáo:

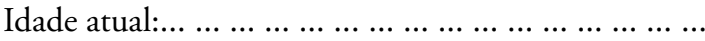

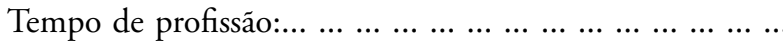

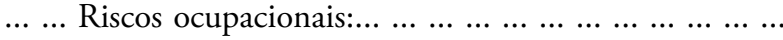
...........

Admissional ( ) Periódico ( ) Retorno ( ) Mudança de Função ( ) Demissional ( )

EXAME FÍSICO GERAL - Data

-Você já sofreu algum acidente de trabalho? Sim ( ) Não ( )

Que tipo?

Comunicou à sua chefia? Sim ( ) Não ( )

-Você já esteve afastado do trabalho por motivo de doença? Sim ( ) Não ( )

$\begin{array}{lllllllllllllllll}\text { Qual } & \ldots & \ldots & \ldots & \ldots & \ldots & \ldots & \ldots & \ldots & \ldots & \ldots & \ldots & \ldots & \ldots & \ldots & \ldots & \ldots\end{array}$ Por quanto tempo? $\ldots \ldots \ldots \ldots \ldots \ldots \ldots \ldots \ldots \ldots \ldots$

Teve alguma complicação em sua recuperação? Sim ( ) Não ( )

-Atualmente, você sente algum desconforto provocado por aquele acidente ou doença?

Sim ( ) Não ( )

-Existe alguma coisa em seu ambiente de trabalho que the proporcione algum desconforto? Sim ( ) Náo ( )

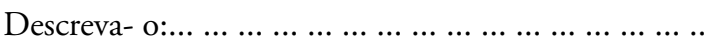

- Tem reaçáo alérgica por algum produto de seu ambiente de trabalho? Sim ( ) Não ( )

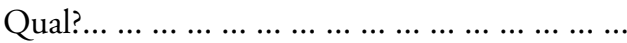

- Sente tontura no ambiente de trabalho?

Sim ( ) Não ( )

- Sente falta de ar no seu ambiente de trabalho? Sim ( ) Não ( )

- Sua boca fica ressecada no ambiente de trabalho?

Sim ( ) Não ( )

- Desde o ultimo exame odontológico periódico, você fez alguma cirurgia? Sim ( ) Não ( )

Qual?...

- Você está fazendo algum tratamento médico atualmente? Sim ( ) Não ( )

Qual?..

- Você esta fazendo algum tratamento odontológico atualmente? Sim ( ) Não ( )

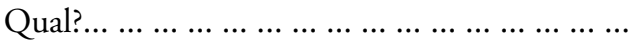

- Você tem algum tipo de dor? Sim ( ) Não ( )

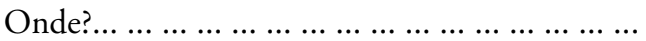

- A dor melhora com alguma coisa? Sim ( ) Não ( ) Em caso afirmativo, como melhora?

- A dor piora com alguma coisa? Sim ( ) Não ( ) Em caso afirmativo, descreva como:

- Você fez algum tratamento para esse problema? Sim ( ) Não ( ) Melhorou? Sim ( ) Não ( )

- Você usa Prótese ? Sim ( ) Não（ ）

- De que tipo :

- Onde costuma guardá-la, caso a retire, em seu trabalho ou na sua casa:

- Você gostaria de mencionar alguma informação sobre sua saúde? Sim ( ) Não ( )

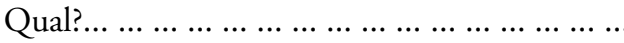

Declaro que as informaçôes fornecidas são verdadeiras.

Data , _____ _ _ _ _ _

Assinatura do funcionário 
Olival ARB, Charone S, Groisman S. A importância do exame odontológico periódico ocupacional: uma proposta de prontuário odontológico. Revista de Odontologia da Universidade Cidade de São Paulo 2008 jan-abr; 20(1):37-45

\section{EXAME DENTAL - DESCRIÇÃO DENTE - A - DENTE}

Data:

18

17

16

$15(55)$

14 (54)

$13(53)$

12 (52)

$11(51)$

21 (61)

22 (62)

23 (63)

24 (64)

25 (65)

26

27

28

38

37

36

35 (75)

34 (74)

33 (73)

$32(72)$

$31(71)$

41 (81)

42 (82)

43 (83)

44 (84)

45 (85)

46

47

48 


\section{REFERÊNCIAS}

1. Conselho Federal de Odontologia: Código de ética odontológica, Rio de Janeiro: CFO, 2004.

2. Cottone JA, Standish SM. Outtline of forensic dentistry. Chicago: Year Book Medica, 1982. P.177

3. Domingues JMM. Exame médico periódico $[\mathrm{Mo}-$ nografia na Internet]. Campo Grande, MS, 2002. [Acesso em: ago 2005] Disponivel em: http://www. fundacentro.sc.gov.br/areas/8.pdf

4. Guimarães E, Rocha AA .Odontologia do trabalho: organização dos serviços odontológicos de uma empresa $1^{\text {a }}$ parte. Odontol Mod 1979; 6(7):7-12

5. Gomes EB, Magalhães H. Censo bucal na empresa: sua importância no planejamento da assistência odontológica. Odontól Mod 1980; 7(3):31-8.

6. Mealey BL. Periodontal implications: medically compromised patients. Ann Periodont 1:307, 1996.
7. Oliveira RN, Daruge E. Contribuição da odontologia legal à identifica [Acesso em: dez 1999. Disponível em: www.cro.com.br/rogerio/contribuicaool. htm (atualmente em www.ibemol.com.br)]

8. Ramirez AIC. Legislacion y odontologia. Dentista y paciente, 1992; 1(8):12-4

9. Silva M, Ramos D, Maruyama N. Compêndio de odontologia legal: alguns comentários sobre ética profissional odontológica. São Paulo: Medsi, 1997. p.53,54,67,68.

10. Zimmermann RD, et al. A importância do prontuário odontológico. Revista do CRO/PE, Recife, 1998; Abr; 1(1).

Recebido em:24/05/2006

Aceito em: 06/05/2007 